\author{
Associate Professor, Wei-Jie ZHOU, PhD \\ E-mail: wjzhou@cczu.edu.cn. \\ Business College, Changzhou University, Jiangsu Changzhou, China \\ Xiao-Li WU, Postgraduate \\ Changzhou University, Jiangsu Changzhou, China \\ Jiao PAN, Postgraduate \\ Changzhou University, Jiangsu Changzhou, China \\ Professor Zheng-Xin WANG, PhD \\ School of Economics, Zhejiang University of Finance \& Economics \\ Hangzhou, China
}

\title{
RECURRENCE INTERVALS ANALYSIS OF CSI 300 FUTURE BASED ON HIGH FREQUENCY DATA
}

\begin{abstract}
Based on 1-minute high frequency close prices of CSI300 future, the statistical properties of volatility and trading volume recurrence intervals exceeding different thresholds are studied in this paper. The result shows that the probability density curves of two recurrence intervals exhibit scaling features. A Weibull function can be used to fit the tail in two distribution curves of scaled recurrence intervals. The memory with short and long term is verified in two recurrence intervals by conditional probability density and detrended fluctuation analysis (DFA), which indicate that the recurrence intervals can be predicted. Using the multifractal detrended fluctuation analysis (MFDFA), we discover that two recurrence intervals possess multifractality. In addition, the relationship between volatility and trading volume recurrence intervals is analyzed.It is shown that the appearance of large volatility and large volume is synchronous. Finally, we discuss the application of recurrence intervals.

Keywords: Recurrence intervals analysis; Scaling features; Probability density function; Memory; Multifractality Volatility-volume recurrence intervals analysis.
\end{abstract}

JEL Classification: G12, G14, G15

DOI: 10.24818/18423264/54.2.20.18 
Wei-Jie Zhou, Xiao-Li Wu, Jiao Pan, Zheng-Xin Wang

\section{Introduction}

The extreme events in financial markets appeared more frequently in recent years, such as the Asian financial crisis in 1997 and the global financial crisis in 2007. Apparently, understanding the rule in the occurrence of extern events is very important to risk management. New nonlinear analysis methods are employed in financial markets for its similarity of natural system, such as fractals and long-term memory, which are utilized to mark the natural system features originally(Kantelhardt et al., 2002; Guo, et al., 2012; Fantazzini, 2016; Lahmiri, 2017). Nowadays, the research on statistical properties of recurrence intervals attracts much attention (Bunde et al., 2007; Zhao et al., 2016; Xia et al., 2016). Yamasaki et al. (2005) found that there are scaling feature in recurrence intervals distribution of seven typical stocks and currency exchange data, and memory with short and long term was observed in recurrence intervals sequence. Wang et al. $(2006,2007)$ found that stretched exponential function can be used to fit the tail of scaled recurrence intervals distribution in stock, commodities, interest rates, and currencies markets. The further investigation by Wang et al. $(2008,2009)$ indicated that there was multi-scaling behavior in the recurrence intervals distribution, and it originated from capitalization, risk, number of trades, and return factors. Through analyzing recurrence intervals of realized volatility in Shanghai stock market, Ren et al. (2009a, 2009b, 2010a) found that the recurrence intervals distribution showed a good scaling behavior, and a memory with short and longterm was presented in realized volatility recurrence intervals. Moreover, Ren et al. (2010b) studied positive and negative returns recurrence intervals sequence based on the 1-minute data set, and found that there are symmetrical features and Power-law tails displayed in two recurrence intervals distributions. However, Xie et al. (2014) found that there were no scaling behaviors in recurrence intervals by analyzing the energy futures volatility, which is different from the above conclusions.

The Chinese stock index 300 future abbreviated as CSI 300 future is the first financial future in China future markets. According to the industries stocks traded in Shanghai and Shenzhen stock markets, the CSI 300 is a stock market index designed to reflect the overall features of Chinese stock market. The CSI 300 future makes up unilateral mechanism for the stock index, and is widely concerned by the researchers and practitioners. Thus, the risk features of the CSI 300 mean a lot to general market investors and stock future index investors. For the objectivity of risk, if the investors or risk managers have a good command of the recurrence 
Recurrence Intervals Analysis of CSI 300 Future Based on High Frequency Data

intervals, the risk will be alleviated.

Whether the volatility and trading volume recurrence intervals in CSI 300 market have scaling behavior and memory? In this paper, statistical and nonlinear methods are used to analyze the volatility and trading volume recurrence intervals of CSI 300 future.The rest of this paper is showed as follows, section 2 introduces the data description; section 3 gives the probability density function; section 4 studies the memory with short and long term in two recurrence intervals; section 5 investigates the relationship between two recurrence intervals; and the conclusion is discussed in section 6 .

\section{Data}

We select 1-minute prices of CSI 300 future from wind Information co., Ltd for empirical research. The studied period is selected from 16 April 2010 to 31 August 2015, including 1284 trading days.

The volatility is defined as the absolute of logarithmic price return between two consecutive minutes, that is,

$$
R(t)=\left|\ln \left(P_{t}\right)-\ln \left(P_{t-1}\right)\right|, t=1,2, \cdots, N .
$$

Where $N$ is the length of sequence, $N=346680$. In sample set, the CSI 300 future trades from 9:15 to 11:30 in the morning and from 1:00 to 3:15 in the afternoon, so there is a total of 270 minutes transactions in one trading day. The $V(t)$ denotes the trading volume corresponding to the volatility. In many stock markets, the volatility and trading volume have intraday pattern, i.e., U-shaped or L-shaped pattern. This pattern should be removed, or the recurrence intervals will show daily periodicity phenomenon. The intraday pattern of volatility and volume are defined as

$$
\begin{aligned}
& M R(s)=\frac{\sum_{i=1}^{n} R^{i}(s)}{n} \\
& M V(s)=\frac{\sum_{i=1}^{n} V^{i}(s)}{n}
\end{aligned}
$$

DOI: 10.24818/18423264/54.2.20.18 
The $R^{i}(s)$ and $V^{i}(s)$ represent the volatility and trading volume at moment $s$ of day $i$, respectively. The $n$ is the total of trading days, i.e., $n=1284$. As presented in the Figure1, the volatility and trading volume have intraday pattern, the volatility intraday pattern is L-shaped, and the volatility is large at the beginning of trading, and slightly shocks between 11:30 closing and 13:00 opening. For trading volume, the intraday pattern exhibits U-shaped. In order to avoid the daily periodicity phenomenon, intraday pattern should be removed by

$$
\begin{aligned}
R^{\prime}(t) & =\frac{R(t)}{M R(t)} \\
V^{\prime}(t) & =\frac{V(t)}{M V(t)}
\end{aligned}
$$

Then, standardizing the volatility and trading volume by their standard deviations

$$
\begin{gathered}
r(t)=\frac{R^{\prime}(t)}{\sqrt{\left\langle R^{\prime}(t)^{2}\right\rangle-\left\langle R^{\prime}(t)\right\rangle^{2}}} \\
v(t)=\frac{v^{\prime}(t)}{\sqrt{\left\langle v^{\prime}(t)^{2}\right\rangle-\left\langle v^{\prime}(t)\right\rangle^{2}}}
\end{gathered}
$$

The $\langle$.$\rangle symbol implies the sequence mean. The volatility recurrence interval is$ defined as the time interval of two successive volatilities exceeding threshold $q$

$\tau_{q}(i)=t(i \mid r>q)-t(i-1 \mid r>q), i=2,3, \cdots$

Similarly, the recurrence interval of trading volume is

$$
\tau_{Q}(i)=t(i+1 \mid v>Q)-t(i \mid v>Q), i=2,3, \cdots
$$


Recurrence Intervals Analysis of CSI 300 Future Based on High Frequency Data
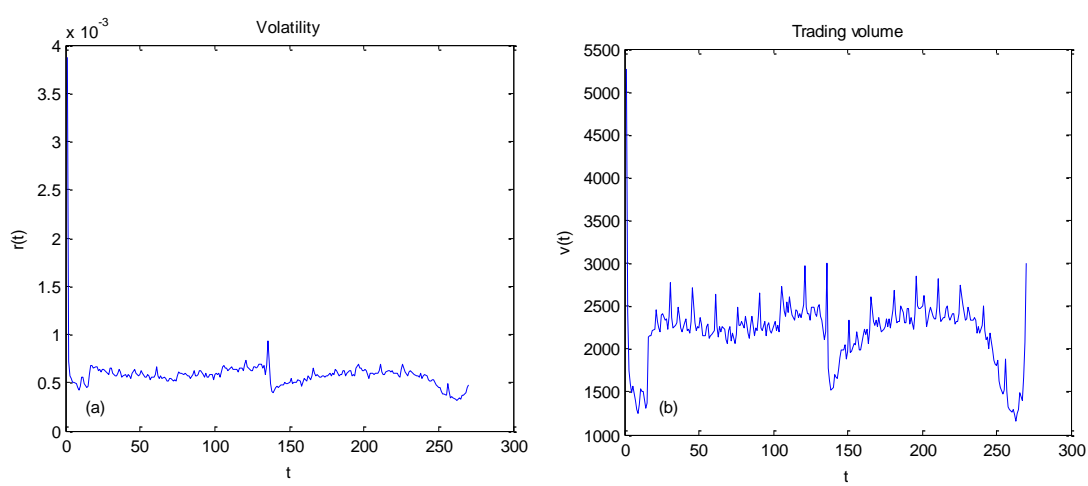

Figure1.Intraday patterns of volatility and trading volume of stock index futures

Figure 2(a) gives the volatility recurrence intervals of different thresholds $q$. The threshold set $q=\{2,3,4,5,6\}$ is selected in this paper. The double logarithmic figure of $q$ and recurrence intervals mean $<\tau_{q}>$ is illustrated in Figure2(b), whose curve can be fitted by $\left\langle\tau_{q}>=0.73 q^{2.53}\right.$. The mean recurrence intervals $\left\langle\tau_{q}\right\rangle$ increases with the increase of threshold $q$. The similar relation between $\left\langle\tau_{Q}\right\rangle$ and $Q$ can be obtained, i.e., $\left\langle\tau_{Q}>=1.14 Q^{2.55}\right.$. For the simplicity, set the $\langle\tau\rangle=\left\langle\tau_{q}>\right.$ or $\langle\tau\rangle=\left\langle\tau_{Q}>\right.$ in this paper. 


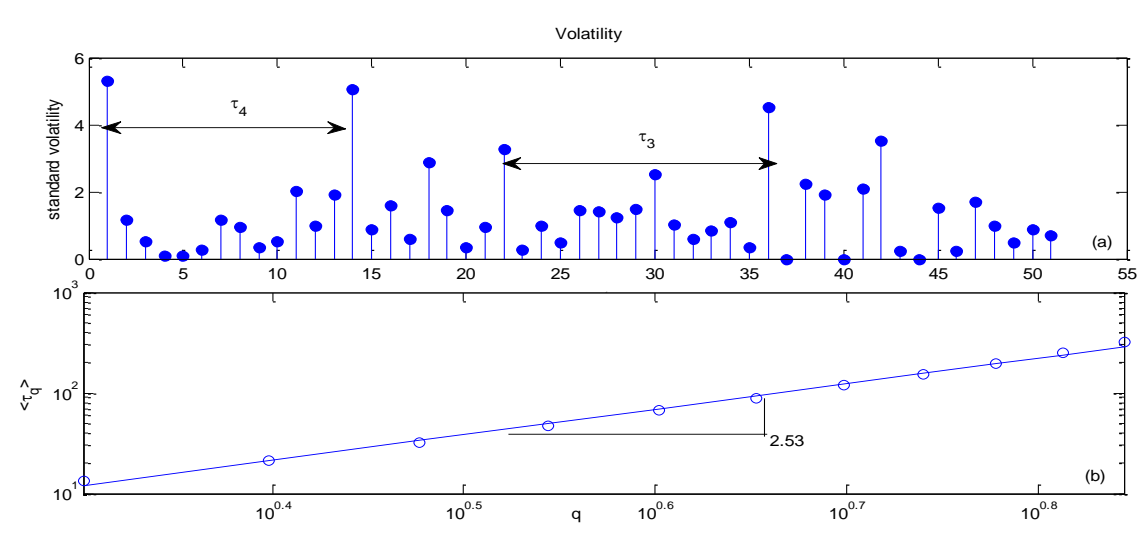

Figure 2. Volatility recurrence intervals and the relationship between $<\tau_{q}>$ and $q$.

\section{The probability density function of recurrence intervals}

The probability distribution of volatility and trading volume recurrence intervals are illustrated in Figure3 (a) and (c). It seems that there is no regularity in probability density curves. However, we found that $P_{q=2}(\tau)>P_{q=3}(\tau)>P_{q=4}(\tau)>P_{q=5}(\tau)>P_{q=6}(\tau)$ for the head of probability densities and $P_{q=2}(\tau)<P_{q=3}(\tau)<P_{q=4}(\tau)<P_{q=5}(\tau)<P_{q=6}(\tau)$ for the tail in probability density curves. Two factors can account for this phenomenon. Firstly, compared to small threshold $q$, the number of recurrence intervals with big threshold is fewer at the head of probability density curves. Secondly, the number of recurrence intervals with big threshold is more than recurrence intervals with small threshold at the tail of probability density curves. As we known, the recurrence intervals mean $\langle\tau\rangle=\left\langle\tau_{q}\right\rangle$ become larger as the threshold value bigger. Therefore, we normalize the recurrence intervals by its mean, i.e., $\langle\tau\rangle$, and plot the scaled probability density curves $\left(P_{q}(\tau)<\tau>\right)$ in Figure3(b) and (d). It is found that there are scaled behaviors in probability density curves of two recurrence intervals, i.e., 
Recurrence Intervals Analysis of CSI 300 Future Based on High Frequency Data

$$
\begin{aligned}
& P_{q}(\tau)<\tau>=f\left(\tau_{q} /<\tau>\right) \\
& P_{Q}(\tau)<\tau>=g\left(\tau_{Q} /<\tau>\right)
\end{aligned}
$$

where $f$ and $g$ are the scaling functions, which are independent of threshold $q$. So, if probability density function (PDF) of recurrence intervals for a threshold $q$ is known, other PDFs of recurrence intervals with different thresholds $q$ can be deduced by $\left\langle\tau_{q}>=0.73 q^{2.53}\right.$ and Eq.(10) or $\left\langle\tau_{Q}>=1.14 Q^{2.55}\right.$ and Eq.(11). Especially, it is important for extreme events with less data by deriving PDFs from smaller threshold $q$.
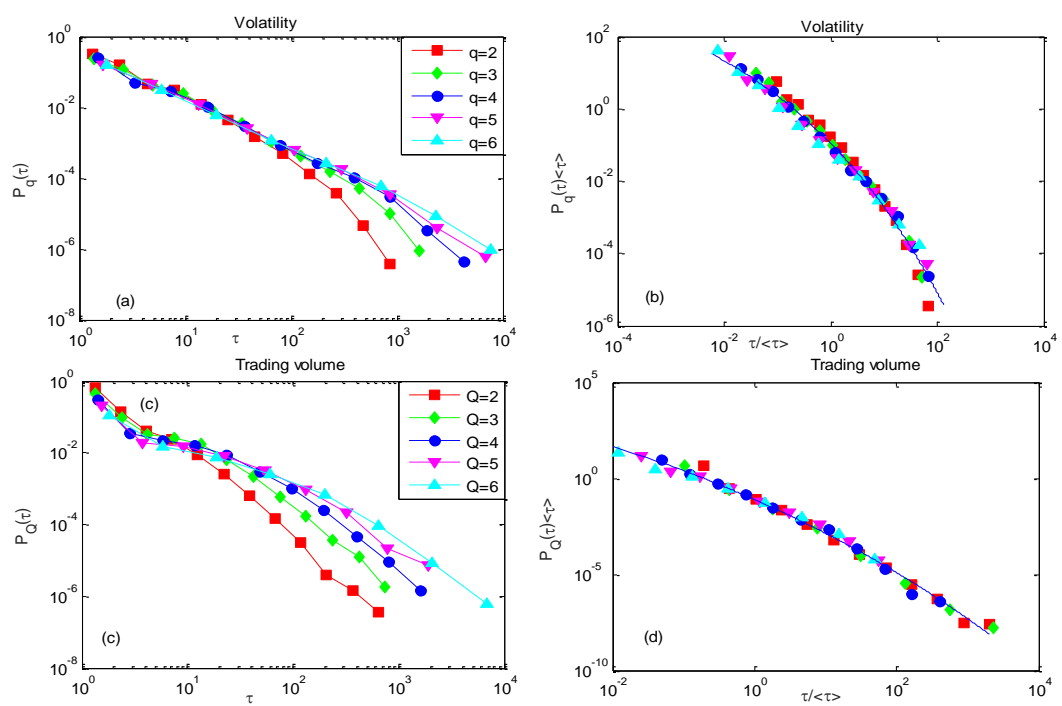

Figure3. Probability and the scaled probability density curves of recurrence intervals

Several empirical studies show that the Power-law or Weibull function can be adopted as the scaling function (Zhao et al., 2016; Ren and Zhou, 2010).

$$
\begin{aligned}
& f(\tau /<\tau>)=f(x)=c x^{-\delta}, x \geq x_{\text {min }} \\
& \quad f(\tau /<\tau>)=f(x)=\alpha \beta x^{\beta-1} \exp \left(-\alpha x^{\beta}\right), x \geq x_{\text {min }}
\end{aligned}
$$


Wei-Jie Zhou, Xiao-Li Wu, Jiao Pan, Zheng-Xin Wang

where $x_{\min }$ is the minimum used in fitting the scaling function. The lowest $x_{\min }$ and parameters are estimated by a method put forward by Clauseet al.(2009). If the PDF of different thresholds $q$ obey the scaling behavior, the PDF curves should all collapse on to a single curve. We use the Kolmogorov-Smirnov (KS) method to test this consistency, and select the optimal fitting between Power-law and Weibull functions (Ren and Zhou, 2010).

Table 1 and 2 give the estimated $\hat{x}_{\min }$, parameters $\delta, c, \alpha, \beta$ and $p$-values of goodness for the Power-law and Weibull fitting. When $p>0.1$, the fitting function can be adopted. For two recurrence intervals, the $\hat{x}_{\min }$ of Power-law fitting are bigger than that of Weibull fitting, which denotes that the scaling range in Weibull fitting is wider. The $p$-values of two recurrence intervals in Power-law fitting are almost close to 0 , while 0.82 and 0.83 in Weibull fitting, demonstrating that recurrence intervals can be fitted by the Weibull function appropriately. Thus, we can use time series model with the Weibull distribution to simulate and forecast the recurrence intervals.

Table 1.The KS test of the volatility and trading volume interval distributions by Power-law fitting

\begin{tabular}{|c|c|c|c|c|c|}
\hline & $\hat{x}_{\min }$ & $\delta$ & $c$ & $K S$ & $p$ \\
\hline Volatility & 0.5943 & 1.4292 & 0.42113 & 0.0524 & 0.0000 \\
\hline $\begin{array}{c}\text { Trading } \\
\text { volume }\end{array}$ & 6.4142 & 1.4180 & 0.4112 & 0.0691 & 0.0000 \\
\hline
\end{tabular}

Table 2.The KS test of the volatility and trading volume interval distributions by Weibull fitting

\begin{tabular}{|c|c|c|c|c|c|}
\hline & $\hat{x}_{\min }$ & $\alpha$ & $\beta$ & $K S$ & $p$ \\
\hline Volatility & 0.0078 & 0.7054 & 0.3685 & 0.0625 & 0.8200 \\
\hline $\begin{array}{c}\text { Trading } \\
\text { volume }\end{array}$ & 2.0452 & 0.1973 & 0.2876 & 0.0707 & 0.8300 \\
\hline
\end{tabular}


Recurrence Intervals Analysis of CSI 300 Future Based on High Frequency Data

\section{The memory of recurrence intervals}

If recurrence intervals sequence has the short or long term memory, we can build the series model for simulating and predicting the recurrence intervals. So, it is interesting to study whether the recurrence intervals sequence possesses memory.

\subsection{The short-term memory}

The conditional probability density function (CPDF) $P_{q}\left(\tau \mid \tau_{0}\right)$ is used to study recurrence intervals sequence with short term memory. The $P_{q}\left(\tau \mid \tau_{0}\right)$ is named as the probability of interval $\tau$ conditioned on its proceeding interval $\tau_{0}$. If $P_{q}\left(\tau \mid \tau_{0}\right)=P_{q}(\tau)$, there is no correlation between the interval $\tau$ and its proceeding interval $\tau_{0}$,i.e., no short term memory. The CPDF of recurrence intervals sequence is investigated by $\tau_{0}$ bins. We rearrange the recurrence intervals sequences with ascending order, and separate into four bins $W_{1}, W_{2}, W_{3}$, and $W_{4}$, among which $W_{1}$ and $W_{4}$ are the smallest and biggest bins respectively. Then, CPDFs $P_{q}\left(\tau \mid \tau_{0}\right)$ of volatility and trading volume recurrence intervals are plotted in Figure4. The $P_{q}\left(\tau \mid W_{1}\right)$ and $P_{q}\left(\tau \mid W_{4}\right)$ are token as filled and open symbols respectively. We take volatility recurrence intervals as an example to illustrate recurrence intervals with short memory. It is shown that $P_{q}\left(\tau \mid W_{1}\right)>P_{q}\left(\tau \mid W_{4}\right)$ for smaller $\tau_{q} /\langle\tau\rangle$, and $P_{q}\left(\tau \mid W_{4}\right)>P_{q}\left(\tau \mid W_{1}\right)$ for larger $\tau_{q} /\langle\tau\rangle$. In other words, small (large) recurrence intervals are more likely to follow such a rule as small (large) recurrence intervals, suggesting the shortterm memory existing in two recurrence intervals sequences. Therefore, clustering also occurs at recurrence intervals, similar to volatility. Similar results about trading volume recurrence intervals can be obtained from Figure4 (b).

DOI: 10.24818/18423264/54.2.20.18 

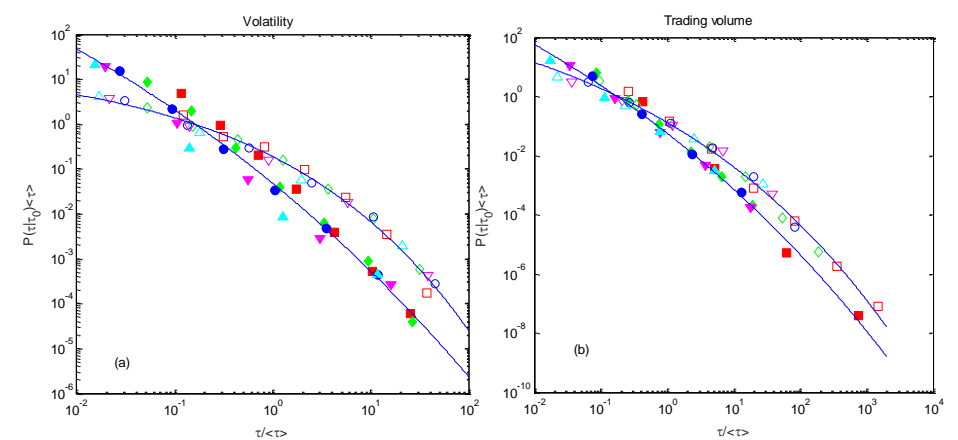

Figure4. Conditional probability density of the smallest set $M_{1}$ (filled symbols)and the largest set $M_{4}$ (open symbols)

\subsection{The long-term memory and multifractality}

We study long term memory of recurrence intervals based on the DFA method advanced by Peng et al.(1994). The DFA method is as follows. Step1. Removing the $n(=1,2)$ order polynomial trend in a window of $s$ points.Step2. Calculating the detrended fluctuation function $F(s)$ and $F(s) \propto s^{H}$. Step3. Analyzing the correlation by the Hurst index $H$. The series has persistently long-term correlated as $0.5<H<1$, and uncorrelated as $H=0.5$, i.e., a random walk. The fluctuation $F(s)$ of recurrence intervals is illustrated in Figure5 (a). It is shown that all fluctuations $F(s)$ are nearly all consistent, indicating that $H$ are fundamentally equivalent in all recurrence intervals. Based on the $F(s) \propto s^{H}$ from Step 3, all $H$ are greater than 0.5 for all recurrence intervals, seen in Table 3 . The results show long term memory existing in the volatility recurrence intervals. Similar results are obtained from trading volume recurrence intervals. Shuffling the volatility sequence, seen in Figure5 (b) and Table 3, the Hurst indexes of all recurrence intervals present closely to 0.5 , suggesting the longterm memory in volatility recurrence intervals may derive from long term memory of volatility. 
Recurrence Intervals Analysis of CSI 300 Future Based on High Frequency Data
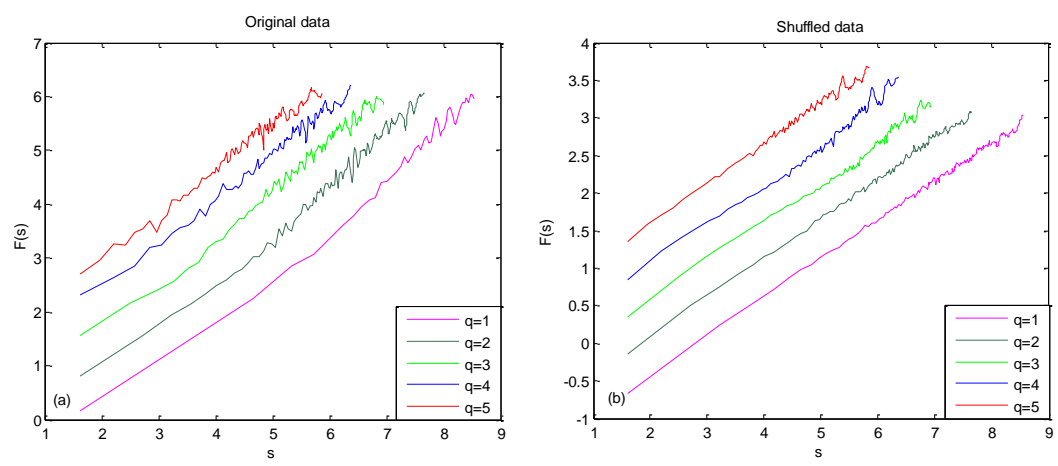

Figure5.Detrended fluctuation function of original and shuffled recurrence intervals

Table 3.Hurst index of volatility and trading volume recurrence intervals

\begin{tabular}{|c|c|c|c|c|c|c|c|c|c|c|}
\hline \multirow{2}{*}{$\mathrm{q}$} & \multicolumn{5}{|c|}{ volatility } & \multicolumn{5}{|c|}{ trading volume } \\
\cline { 2 - 11 } & 2 & 3 & 4 & 5 & 6 & 2 & 3 & 4 & 5 & 6 \\
\hline Original & 0.92 & 0.93 & 0.87 & 0.84 & 0.81 & 0.81 & 0.82 & 0.8 & 0.82 & 0.8 \\
\hline Shuffled & 0.5 & 0.52 & 0.54 & 0.53 & 0.52 & 0.52 & 0.53 & 0.49 & 0.49 & 0.52 \\
\hline
\end{tabular}

We study the multifractality of volatility and trading volume recurrence intervals by multifractal detrended fluctuation analysis(MFDFA) (Kantelhardt et al., 2002), whose algorithm is as follows, the Step 1 is as the same as DFA. Step2. Calculating the $p$-th order fluctuation function $F_{p}(s)$ and the generalized Hurst index $h(p)$ by $F_{p}(s) \square s^{h(p)}$. Step3. Calculating the scaling exponents $\tau(p)$ using $\tau(p)=p h(p)-1$, if $h(p)$ or $\tau(p)$ is a nonlinear function of $p$, the time sequence possesses multifractality. Step4. Obtaining singularity strength $\alpha$ and singularity spectrum $f(\alpha)$ by $\alpha=h(p)+p h^{\prime}(p)$ and $f(\alpha)=p[\alpha-h(p)]+1$. The broader the singularity spectrum, the more complex the multifractality. As seen in Figure6, two recurrence intervals have multifractal feature. Except $Q=2$ and

DOI: 10.24818/18423264/54.2.20.18 
$Q=3$, all recurrence intervals are almost the same $\square \alpha$, where $\square \alpha=\alpha_{\text {max }}-\alpha_{\text {min }}$.

So, multifractality should be considered in modeling the recurrence intervals sequence.
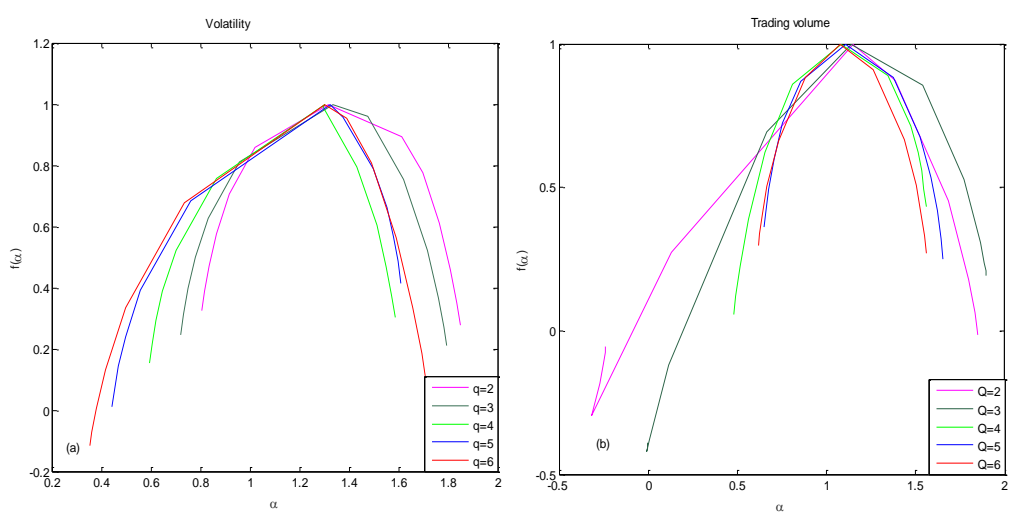

Figure6.Multifractality of volatility and trading volume recurrence intervals

\section{The relationship between volatility and trading volume recurrence intervals}

Figure7 plots the volatility recurrence intervals sequence and its corresponding volume recurrence intervals. It is shown that the volatility recurrence intervals are consistent with trading volume recurrence intervals. Thus, we investigate the relationship of two recurrence intervals sequences by statistical methods. We calculate the conditional probability that the trading volumes have the same recurrence intervals as that of volatility. Set

$$
P\left(\tau_{Q} \mid \mathrm{r}>\mathrm{q}\right)=\frac{N_{v>Q, \tau_{q}}}{N_{\tau_{q}}}
$$

where $N_{\tau_{q}}$ is the number of volatility recurrence intervals with threshold $q$, and $N_{v>Q, \tau_{q}}$ is the number of trading volume recurrence intervals $\tau_{Q}$ condition on two consecutive volatilities exceeding the threshold $q$, i.e., $v(t)>Q$ and $v\left(t+\tau_{q}\right)>Q$ 
Recurrence Intervals Analysis of CSI 300 Future Based on High Frequency Data

when $r(t)>q$ and $r\left(t+\tau_{q}\right)>q$. The $P\left\{\tau_{Q} \mid r>q\right\}$ measures the possibility of volatility recurrence intervals equals trading volume recurrence intervals synchronously. Similarly, the conditional probability $P\left\{\tau_{q} \mid v>Q\right\}=\frac{N_{\tau_{q}, v>Q}}{N_{\tau_{q}}}$ can be defined based on trading volume recurrence intervals with threshold $Q$.

Clearly, $P\left(\tau_{q} \mid v>Q\right)=1$ as $q=0$ and $P\left(\tau_{q} \mid r>q\right)=1$ as $Q=0$.
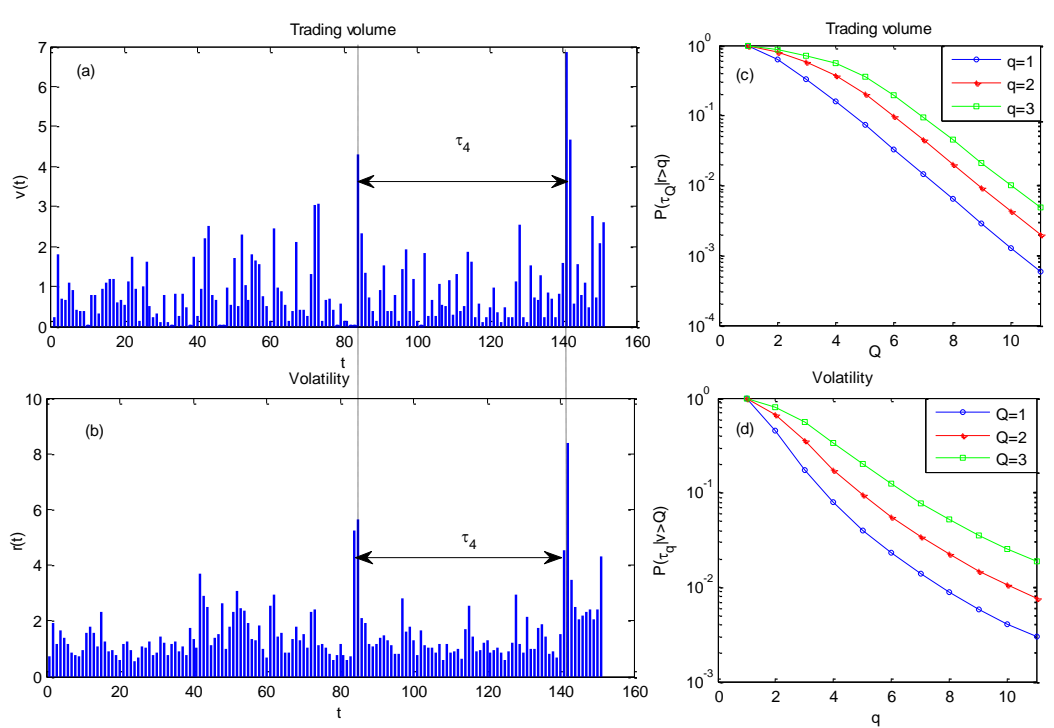

Figure7.The relation between volatility and trading volume recurrence intervals

As illustrated in Figure7(c), the conditional probability of volatility and trading volume recurrence intervals increase when volatility threshold $q$ increases. Similar conclusion is deduced by the probability plot of $P\left(\tau_{q} \mid v>Q\right)$, shown in Figure7 (d). This result suggests that the larger the thresholds $q$ and $Q$, the higher the synchronous equality of two recurrence intervals. While the large threshold $q$

DOI: 10.24818/18423264/54.2.20.18 
Wei-Jie Zhou, Xiao-Li Wu, Jiao Pan, Zheng-Xin Wang

and $Q$ mean that volatility and trading volume are large, implying that large volatility and volume are synchronous in time.

\section{Conclusions}

Based on the statistical and nonlinear methods, this paper analyzes the characteristics of volatility and trading volume recurrence intervals of CSI 300 future. The following conclusions are obtained,

1). Before the research on recurrence intervals, it is only known that the volatility possesses the clustering phenomenon, i.e., large (small) volatility is easier to follow large (small) volatility, but how long to appear next is unknown. The recurrence intervals analysis provides a way to understand its inherent mechanism.

2). The probability density of two recurrence intervals for different thresholds have a scaling behavior. Thus, the probability of extreme events can be deduced by normal events, i.e., the volatility recurrence intervals with threshold smaller $q$.

$3)$. There is a memory with short and longterm in the volatility and volume recurrence intervals, indicating the recurrence intervals can be forecasted. Considering the similarity between recurrence intervals and duration sequences, the Autoregressive Conditional Duration model (ACD) model canbe employed to construct the recurrence intervals sequence. Besides, the volatility and trading recurrence intervals have multifractality, so the multi-scale feature should be added to ACD model.

4). The co-movement between large trading volumes and large volatility is verified by analyzing the volatility and trading volume recurrence intervals. In future, we will study the dependence of trading volumes on volatility, and the risk estimation by recurrence intervals in financial markets.

\section{ACKNOWLEDGEMENTS}

This research was supported by a Marie Curie International Incoming Fellowship within the 7th European Community Framework Programme (Grant No. FP7-PIIF-GA-2013-629051), and the National Natural Science Foundation of China (Grant No. 71701024). 
Recurrence Intervals Analysis of CSI 300 Future Based on High Frequency Data

\section{REFERENCES}

[1]Bunde, A., Eichner, J.F., Kantelhardt, J.W.,Havlin, S.(2007),Long-Term Memory: ANatural Mechanism for the Clustering of Extreme Events and Anomalous Residual Times in Climate Records.Phys. Rev. L, 99, 240601; [2] Clauset, A., Shalizi, C.R., Newman, M.E.J.(2009), Power-law Distributions in Empirical Data.SIAM Rev, 51(4), 661-703;

[3] Fantazzini D. (2016), The Oil Price Crash in 2014/15: Was there a (Negative) Financial Bubble?.Energy Policy, 96, 383-396;

[4] Guo, K., Zhou, W. Cheng, S. (2012),Economy Barometer Analysis of China Stock Market: A Dynamic Analysis Based on the Thermal Optimal Path Method. Journal of management sciences in China, 15(1), 1-10;

[5] Kantelhardt, J.W., Zschiegner, S.A., Koscielny, B.E., Havlinda, S., Bundea, A., Stanley, H.E. (2002), Multifractal Detrended Fluctuation Analysis of Nonstationary Time Series.Physica A, 316, 87-114;

[6] Ludescher, J., Bunde,A. (2014),Universal Behavior of the Interoccurrence Times between Losses in Financial Markets: Independence of the Time Resolution. Physical Review E,90(6), 062809;

[7] Lahmiri, S. (2017),Multifractal Analysis of Moroccan Family Business Stock Returns.Physica A, 486,183-191;

[8] Peng, C.K., Buldyrev, S.V., Simons, M., Havlin, S., Stanley, H.E., Goldberger, A.L. (1994),Mosaic Organization of DNA Nucleotides. Physical Review E, 49,1685-1689;

[9] Ren, F., Gu, G., Zhou, W. (2009),Scaling and Memory in the Return Intervals of Realized Volatility.Physica A, 388, 4787-4796;

[10] Ren, F., Guo, L., Zhou, W. (2009), Statistical Properties of Volatility Return Intervals of Chinese Stocks. Physica A, 388, 881-890;

[11] Ren, F., Zhou, W. (2010), Recurrence Interval Analysis of Trading Volumes. Phys. Rev. E, 81,066107;

[12] Ren, F., Zhou, W. (2010), Recurrence Interval Analysis of High-Frequency Financial Returns and Its Application to Risk Estimation. New Journal of Physics, 12, 075030;

[13] Wang, F., Kazuko,Y., Shlomo, H., Stanley, H.E. (2006),Scaling and Memory of Intraday Volatility Return Intervals in Stock Markets. Phys. Rev. E, 73, 026117;

DOI: 10.24818/18423264/54.2.20.18 
Wei-Jie Zhou, Xiao-Li Wu, Jiao Pan, Zheng-Xin Wang

[14] Wang, F., Weber, P., Yamasaki, K., Havlin, S., Stanley, H.E. (2007),

Statistical Regularities in the Return Intervals of Volatility. Eur. Phys. J. B, 55,

123-133;

[15] Wang, F.,Yamasaki, K., Havlin S., Stanley, H.E. (2008), Indication of

Multiscaling in the Volatility Return Intervals of Stock Markets. Phys. Rev. E, 77, 016109;

[16] Wang, F., Yamasaki K.,Havlin,S., Stanley, H.E. (2009),Multifactor Analysis of Multiscaling in Volatility Return Intervals. Phys. Rev. E, 79, 016103;

[17] Xia, M., Cai, C.S., Pan, F.,Yu, Y. (2016),Estimation of Extreme Structural Response Distributions for Mean Recurrence Intervals Based on Short-Term Monitoring. Engineering Structures, 26, 121-132;

[18] Xie,W., Jiang, Z., Zhou, W. (2014),Extreme Value Statistics and Recurrence Intervals of NYMEX Energy Futures Volatility. Econ. Modell, 36, 8-17;

[19] Yamasaki, K., Muchnik, L., Havlin, S., Bunde A., Stanley, H.E. (2005),Scaling and Memory in Volatility Return Intervals in Financial Markets. PNAS, 102(26),9424-9428;

[20] Zhao, X., Shang, P., Lin, A. (2016),Universal and Non-universal Properties of Recurrence Intervals of Rare Events. Physica A. 448, 132-143. 